Representaciones juveniles sobre violencia de género: significados y contextos

Aldana Florencia Romano, Yussef Becher

Question, Vol. 1, N. 62, e159, abril-junio 2019

ISSN 1669-6581 | https://doi.org/10.24215/16696581e159

http://perio.unlp.edu.ar/ojs/index.php/question

FPyCS | Universidad Nacional de La Plata

La Plata | Buenos Aires | Argentina

\title{
Representaciones juveniles sobre violencia de género: significados y contextos
}

Youth representations about gender violence: meanings and contexts

\author{
Aldana Florencia Romano romanoaldana@gmail.com \\ Universidad Nacional de San Luis (Argentina) \\ Yussef Becher yussefbe@hotmail.com \\ https://orcid.org/0000-0003-1256-3890 \\ Universidad Nacional de San Luis/ Consejo Nacional \\ de Investigaciones Científicas y Técnicas (Argentina)
}

\section{Resumen}

La violencia de género como problemática social ha ido adquiriendo una significativa centralidad en tiempos actuales, lo cual se vincula con la importante visibilización que tuvo desde la década pasada, tanto a partir de las militancias e intervenciones feministas como de 
las políticas de Estado. De allí que el gobierno posterior -más allá de sus fundamentos ideológicos- no duda en recurrir a un discurso que coloca a las desigualdades sociales de género en uno de los primeros puestos de sus rankings, aunque luego ello no se manifieste en políticas públicas.

Diferentes estudios -desde la antropología o desde la sociología- han demostrado la centralidad de instituciones tales como la familia, el empleo o la educación en la reproducción de discursos y comportamientos que develan significativas connotaciones de violencia. Por ello -tal como mencionan los mismos estudios- tiene sentido detenernos a observar los comportamientos de las generaciones juveniles, por cuanto aquellas instituciones tradicionales y sus organizaciones instituidas ejercen un papel central en la transmisión intergeneracional. Para conocer significados y contextos nos interesa profundizar en las representaciones sociales que las juventudes construyen sobre la violencia de género.

Este estudio -que se halla inserto en el proyecto de investigación (PROICO 15-0418) "Juventudes Contemporáneas" de la UNSL- es una primera aproximación al objetivo planteado y para ello acude a una estrategia cualitativa recurrente entre quienes se proponen bucear de modo preliminar en las significaciones sociales de los grupos juveniles: el método asociativo. Los resultados preliminares muestran la predominancia de significantes ligados a la violencia de género física -incluyendo al feminicidio- colocando al patriarcado o las desigualdades como las principales culpables de aquellas violencias. Asimismo, entre los ámbitos en donde mencionan haber padecido situaciones de violencia ubican en primer lugar sus relaciones afectivas de pareja y la universidad o escuela.

Palabras clave: Violencia de género; juventudes; representaciones sociales.

\section{Abstract}

Gender violence as a social problem has been acquiring a significant centrality in present times, which is linked to the important visibility that it has had since the last decade, both from militancy and feminist interventions and from state policies. Hence, the subsequent government -beyond its ideological foundations- does not hesitate to resort to a discourse that places social inequalities of gender in one of the top positions of their rankings, although then this is not manifested in public policies.

Different studies -from anthropology or from sociology- have demonstrated the centrality of institutions such as family, employment or education in the reproduction of discourses and behaviors that reveal significant connotations of violence. Therefore -as the same studies mention- it makes sense to stop and observe the behavior of the youthful generations, because 
those traditional institutions, and their instituted organizations, play a central role in intergenerational transmission. From this, to know meanings and contexts, we are interested in deepening the social representations that youth build on gender violence.

This study -which is inserted in the research project (PROICO 15-0418) "Contemporary Youth" of the UNSL- is a first approximation to the proposed objective and for this it goes to a recurrent qualitative strategy among those who intend know the social meanings of youth groups: the associative method. The preliminary results show the predominance of signifiers linked to physical gender violence -including femicide- placing patriarchy or inequalities as the main culprits of those violence. Likewise, among the areas where they mention having suffered situations of violence, they place first their affective relationships as a couple and the university or school.

Keywords: Gender violence; youths; social representations.

No es sencillo iniciar un texto en Ciencias Sociales por cuanto quienes los escribimos pretendemos satisfacer varias necesidades: por una parte ser rigurosos con el desarrollo posterior y por otra no agobiar a las/os lectores con cuestionamientos o despliegues teóricos grandilocuentes que poco incentivan a continuar la lectura. En tal sentido vamos a plantear algunos de los ejes y análisis con los cuales se van a encontrar quienes se propongan leer este artículo.

En primer lugar vale señalar que el propósito de este texto es bucear en las representaciones sociales juveniles sobre violencia de género. Para dar un primer paso es necesario reconocer que las representaciones se hallan insertas en un contexto social determinado -con los discursos que le son propios y que van incidiendo en las cogniciones y las prácticas- y que, al mismo tiempo, ese contexto se encuentra influenciado por espacios organizados integrados por instituciones. En una segunda instancia debemos reconocer que tales organizaciones instituidas socializan en normas y valores a quienes transitan por ellas. $Y$ en ese tránsito cotidiano transcurren las juventudes, quienes son las disciplinadas predilectas por parte de esas organizaciones. No es casual que el devenir generacional de las/os jóvenes implique el paso por los ritos institucionales de la familia y los ámbitos educativos. En esos espacios, tal parece, las representaciones sociales jugarían un papel relevante por cuanto permitirían construir esquemas de sentido acerca de los modos dominantes de interpretar el mundo social. 
Por consiguiente tales figuraciones simbólicas serían claves para garantizar el flujo de la interacción social e intersubjetiva.

La problemática de la violencia de género ha ido obteniendo un espacio protagónico en los discursos sociales cotidianos, lo cual deriva, en gran medida, del preocupante incremento de los feminicidios, que ha ido motivando movilizaciones y activismos que han logrado instalar el reclamo en las agendas mediáticas y políticas. No nos es difícil recordar la presencia que tuvo el feminicidio de Ángeles Rawson o de Wanda Taddei en los medios de comunicación con notables dejos de espectacularización. Sin embargo, y a pesar de ello, tales presencias mediáticas han ido generando condiciones para poner el asunto en el tapete de los debates cotidianos. De modo tal que han motivado un movimiento -cuya emergencia data de 2015, aunque tiene antecedentes previos- aglutinado bajo el lema de $\mathrm{Ni}$ una Menos, que ha tenido como principal premisa impugnar y denunciar la muerte de mujeres como consecuencia de la misoginia y la ausencia de políticas estatales que intenten contrarrestarla. Con el transcurso del tiempo, el movimiento ha ido mutando e incorporando otros reclamos que -parafraseando a Judith Butler (2007)- permiten superar los esencialismos identitarios femeninos e incorporar otras demandas y por consiguiente a otros/as actores. El último lema fue: Sin \#AbortoLegal no hay \#NiunaMenos. No al pacto de Macri con el FMI.

Por tales motivos nos parece interesante conjugar los aspectos antes planteados y proponer una primera aproximación a las representaciones que las juventudes construyen sobre la violencia de género.

Para ello comenzamos reflejando la presencia social del tema en la Argentina. Continuamos el tejido con un fragmento en donde develamos el modo en que se cosen los conceptos que proponemos para efectuar este primer acercamiento. Seguidamente presentamos los resultados en los que es posible advertir la presencia de sentidos dominantes influenciados por el contexto y el espacio social epocal. Asimismo, para reflejar la presencia de las organizaciones instituidas, a las que antes aludimos, efectuamos el ejercicio analítico de separarlas de los significados, indagando sobre las organizaciones en las que las/os jóvenes consultados manifiestan haber padecido situaciones de violencia de género. Finalmente arribamos a reflexiones que intentan unir -sin esconder los hilos- el tejido que hemos ido construyendo en este texto. No escondemos los hilos pues nos parece ineludible en ciencias sociales reconocer que toda lectura puede ser potenciada a partir de la construcción colectiva que supone la publicación de resultados en espacios que congregan a investigadores/as. 


\section{Precisiones metodológicas}

En el marco del proyecto de investigación "Juventudes Contemporáneas" (FCEJS-UNSL) se llevaron a cabo las actividades de investigación cuyos primeros resultados se presentan en este artículo. Tales actividades consistieron en cuestionarios abiertos de los que participaron jóvenes cuyas edades oscilan entre los 19 y 27 años. Otras particularidades de la muestra son descriptas en el texto.

Consideramos, lo cual introduce peculiaridades metodológicas, que siempre que abordamos el concepto juventudes -tal como plantea Bonvillani (2016)- es necesario dar cuenta de los presupuestos epistemológicos que lo rodean, y agregamos, por nuestra parte, que ello implica una actitud investigativa que permita revelar las condiciones sociales en las que se encuentran insertos los integrantes del colectivo socio generacional. Ello por cuanto al advertir tales condiciones podemos apreciar su incidencia en la construcción social de la edad y por consiguiente de la juventud. El punto Perfiles juveniles tiene esa finalidad.

Para desentrañar las representaciones juveniles sobre violencia de género optamos por el método asociativo por cuanto permite descubrir los principales significantes que rodean la representación sobre un objeto determinado. A partir de ello se pueden conocer los sentidos predominantes y los contextos constitutivos de las figuraciones simbólicas. El cuestionario incorporó -luego de consultar sobre datos que permitieran identificar los perfiles- las siguientes preguntas: 1. Ante el término violencia de género, ¿qué palabra o palabras se le ocurren? Mencionar aquellas que espontáneamente se le vayan ocurriendo. 2. De las palabras que mencionó, defina brevemente el significado que para usted tienen. 3. De todas las palabras que usted mencionó, indique cuál es la que considera más importante en relación con violencia de género. 4. ¿Considera que ha vivido alguna situación de violencia de género? En tal caso, ¿en qué marco se ha producido?

Para el relevamiento de los datos consultamos a jóvenes que transitaban por espacios públicos si estaban dispuestos a completar voluntariamente los cuestionarios. Ante lo cual les mencionamos detalladamente las intenciones del estudio, como así también el proyecto y organismos de ciencia y técnica en los que se inserta. Asimismo, a los fines de preservar la identidad, los cuestionarios fueron anónimos. Si bien -tal como aclaramos posteriormente- no consideramos a la juventud sólo anclada a criterios etarios, para definir la muestra e identificar intencionalmente a las juventudes que podrían participar de esta primera etapa, fue necesario tener en cuenta determinadas edades. De allí que recurrimos a los límites etarios de juventud establecidos por el Instituto Nacional de Estadísticas y Censos (INDEC) o la Organización Iberoamericana de la Juventud (OIJ), que delimitan la etapa juvenil entre los 14 y 29 años. 
Los cuestionarios suman un total de cuarenta casos que fueron seleccionados intencionalmente, en donde buscamos equiparar representación por géneros y diversidad de edades. Sin embargo, la muestra se encuentra constituida mayoritariamente por mujeres y, al mismo tiempo, no pudimos relevar casos de identidades sexuales disidentes. Para analizar los datos construimos una matriz en donde fuimos jerarquizando las palabras más recurrentes, como así también aquellas otras que aparecían con menor frecuencia. Asimismo agrupamos aquellos casos que señalaron haber padecido alguna situación de violencia de género y los espacios $u$ organizaciones en los que mencionan haber experimentado tales situaciones. Para construir las tablas y los gráficos acudimos al sistema de procesamiento de datos Statistical Package for the Social Sciences (SPSS).

\section{Violencia de género: su presencia en la Argentina}

En 1995, Susana Chávez, una poeta de nacionalidad mexicana, escribió las estrofas de Ni una muerta más en las que reflejaba los diversos feminicidios cometidos en Ciudad de Juárez. Aunque denunciar y militar no la mantuvo ajena de la realidad que ella misma impugnaba, en 2011 aparece muerta, el motivo: ser mujer.

La violencia basada en razones de género existe desde los comienzos mismos de la historia pos colonialidad; sin embargo, durante muchos años quienes la han padecido han ocultado tal realidad por miedo a prejuicios sociales y a una evidente falta de acompañamiento gubernamental. De acuerdo con los datos proporcionados por La Casa del Encuentro Organización no gubernamental (ONG) dedicada a dicha problemática-, en la Argentina muere aproximadamente una mujer cada 30 horas. En nueve de cada diez casos de violencia de género, la persona que la sufre está vinculada con su agresor en el marco de una relación de pareja, ya que son autores de las agresiones novios, convivientes, cónyuges y ex parejas. Asimismo, la experiencia de la violencia suele manifestarse en las trayectorias vitales a temprana edad, es decir, en la etapa de la adolescencia y la juventud en la cual quien la padece sufre primero agresiones con secuelas psicológicas y, luego, físicas y en algunos casos sexuales (1). Ante tal realidad, en la Argentina se promovió, luego del reclamo de parte de diversos movimientos feministas, con importantes antecedentes a nivel internacional, que se debata en el seno del Congreso Nacional (2). A partir de ello, el 11 de marzo de 2009 se sancionó la Ley Nro. 26485 denominada De Protección Integral de las Mujeres. Tal normativa define a la violencia contra las mujeres, 
a toda conducta, acción u omisión, que, de manera directa o indirecta, tanto en el ámbito público como en el privado, basada en una relación desigual de poder, afecte su vida, libertad, dignidad, integridad física, psicológica, sexual, económica o patrimonial, como así también su seguridad personal. Quedan comprendidas las perpetradas desde el Estado o por sus agentes (artículo 4) (3).

En el marco de dicha normativa se incorporó al Código Penal una figura calificada como femicidio, a fin de establecer como delitos los homicidios consecuencia de la violencia de género que tienen como destinatarias a las mujeres. Si bien dicho cuerpo normativo incorpora causales generales de justificación o eximición de pena por el ejercicio de la legítima defensa, las interpretaciones legales androcéntricas suelen excluir de sus alcances a las mujeres que, en defensa propia o de sus hijos, resisten los ataques de sus agresores. Ello se vincula con la ligazón entre el derecho y las estructuras patriarcales, pues el derecho como institución, representado en su dispositivo institucional de preferencia: el Poder Judicial (sea a nivel local o nacional), realiza distinciones irracionales entre varones y mujeres. Olsen comenta:

\footnotetext{
los jueces han declarado, por ejemplo, la inconstitucionalidad de leyes que establecían preferencias por los hombres sobre las mujeres, o que establecían que los padres debían mantener a sus hijas hasta una edad menor que sus hijos, o bien de leyes que fijaban diferentes edades -según se tratara de ser hombres y mujeres- para autorizar la compra de bebidas alcohólicas, etc. (2000: 148).
}

Al mismo tiempo, a pesar de la vigencia de estas normativas, los hechos de violencia basados en el género han ido incrementando anualmente de manera progresiva. Por tal motivo comenzaron a visibilizarse manifestaciones masivas contra esas realidades a partir de 2015 aglutinadas bajo el lema Ni una Menos, que reconoce antecedentes en la propuesta de Susana Chávez. Entre los feminicidios que motivaron ese activismo se halla la muerte de una joven de 14 años, Chiara Páez, quien, estando embarazada, fue asesinada por su novio en la localidad de Rufino (provincia de Santa Fe).

Del primer Ni una Menos realizado en la Ciudad de Buenos Aires en la Plaza del Congreso, que fue replicado en otras provincias argentinas, participaron alrededor de 150.000 personas, tal como se reconoce en la web del movimiento. A partir de esta primera manifestación todos los años se reitera, incrementando su masividad y alcance. 
Tanto el plano de lo simbólico como el de lo material ejercen su influencia decisiva en la conformación del concepto juventud(es). Esto ha sido señalado por diferentes investigadores en dicho campo de estudios. Podemos acudir a Mary Jo Maynes (2008) cuando apelando a la historia personal de Adelheid Popp -feminista socialista austríaca- muestra el modo en que se anudan las condiciones materiales de su existencia -que no le permitían autopercibirse como una niña- y la incidencia de lo simbólico por cuanto en el mundo adulto era significada como una adolescente. El concepto de representaciones sociales ha sido recurrentemente utilizado en las investigaciones sobre juventudes. Tradicionalmente se han distinguido dos enfoques en el estudio de dicho concepto de las ciencias sociales: el de Aubric (1976) y el de Moscovici (1993). Ambos confluyen en identificar la relevancia del examen de las representaciones para conocer las matrices de sentido con las cuales los actores sociales conocen el mundo. Por ello tales representaciones son definidas como construcciones discursivas que apelando al sentido social -o a los discursos sociales- logran instalarse como modos de interpretar la realidad. El enfoque de Aubric afirma que buceando en las representaciones sociales se pueden reconocer núcleos de sentido centrales, presentes en las diferentes manifestaciones simbólicas de la figuración significativa. Moscovici señala que mediante el estudio de la representación es posible identificar los elementos periféricos que integran esas versiones del mundo que cada grupo de sujetos construye. Distingue etapas en la conformación de la representación social que devienen en lo que denomina objetivación y anclaje. Ambos conceptos -como nos va a ayudar a desentrañar los estudios posteriores de Jodelet (1986)- reflejan el engranaje que existe entre cogniciones y prácticas. De allí que aquello que conocemos y que estructuramos de acuerdo con nuestros esquemas de sentido -en donde la representación social ejerce su predominio- luego se manifiesta en los comportamientos subjetivos. Sin embargo, aportes posteriores -en donde podemos ubicar el de González Rey (2008)- nos permiten comprender que no sólo los comportamientos están atravesados por la subjetividad, sino que también el modo en que conocemos va a ser influenciado por aquella. Ello nos muestra que los pretendidos conocimientos -que actúan como matrices de sentido sociales tal como lo planteaba Moscovici- en realidad también están impregnados de un halo de subjetividad. A partir de ello que cada sujeto o grupo de sujetos puede conocer y comportarse de un modo distinto a otro a partir de la influencia de la subjetividad. Ello le otorga a la psique un rol más activo que aquel papel residual al cual la habían relegado enfoques freudianos y posfreudianos. Por ello, las perspectivas posestructuralistas -que han incidido en diferentes disciplinas- han ido resignificado el papel de los procesos cognitivos a partir del concepto de subjetividad como una 
construcción social. Guattari (2006) afirma: “... la subjetividad está esencialmente fabricada y modelada en el registro de lo social" (p. 46). En el entramado social se ponen en juego el papel de las instituciones y del espacio social. Toda institución se halla inserta en un espacio social y viceversa: el espacio social es construido por las instituciones. Con el transcurso del tiempo las ciencias sociales han ido reconfigurando el concepto de espacio social, pues no sólo se trata de fronteras geográficas que dividen a uno u otro territorio. Se trata, también, -tal como afirma Doreen Massey (2007)- de una construcción simbólica en donde predominan relaciones de poder que marcan distinciones entre unos y otros sujetos. Más allá del concepto de institución que se tenga en cuenta, todos ellos redundan en definirlas como un conjunto de pautas que constriñen los comportamientos subjetivos. Sin embargo -tal como apunta Dubet (2010)podemos identificar diferentes registros semánticos en la noción de institución. En tal sentido, el autor propone reparar en la función de instituir y socializar que es propia de las organizaciones instituidas, "La institución se define por su capacidad de promover un orden simbólico y formar un tipo de sujeto amoldado a cierto orden, en definitiva, de instituirle" (p. 16). De allí, la posibilidad de reconocer en las instituciones un papel clave en la transmisión intergeneracional por cuanto esos procesos de socialización se cultivan en, "la iglesia, la escuela, la familia o la justicia... porque inscriben un orden simbólico y una cultura en la subjetividad de los individuos, porque "institucionalizan" valores y símbolos..." (p. 16). Todos ellos espacios que son transitados recurrentemente por las juventudes en torno a los cuales -tal como muestra Florencia Saintout (2007) en su tesis doctoral- rondan, no sin cuestionarlos o resignificarlos, reconociendo su valor como espacios de socialización y de construcción de redes sociales. Entonces -retomando el tema del espacio- si las instituciones configuran esa esfera en la cual predominan las relaciones de poder; allí, el género -como elemento clave en la construcción de ese tipo de relaciones- se vuelve un aspecto relevante que vale la pena revisar. Las relaciones sociales de género son una construcción asimétrica que como tal se empapa en la existencia de diferencias biológicas entre los sexos. Los estereotipos de género tal como afirman Cook y Cusack (2010)- construyen guiones acerca de los comportamientos que son apropiados para cada sexo y a partir de ello restringen recursos y derechos. Podemos tomar el sencillo ejemplo -aunque sumamente dañino por sus efectos- que estereotipa a las mujeres como las cuidadoras y reproductoras de la especie, y en ello limita sus posibilidades de experimentar libremente su sexualidad y de mayor involucramiento en la esfera pública. Parafraseando a María Luisa Femenías (2018) podemos aseverar que las opresiones sobre el género responden a un proceso bifronte: sujeciones al orden social establecido e inacción estatal para intentar subvertir dicho orden. Todas esas sujeciones e inacciones finalmente 
constituyen modalidades de violencia -más o menos evidentes- que afectan el pleno desarrollo y ejercicio de derechos por parte de las mujeres.

A partir de los conceptos antes enunciados y puestos en juego reconocemos que los sujetos se construyen -tal como afirmaba Guattari- en el registro de lo social. En ese registro elaboran figuraciones simbólicas que les permiten interpretar el mundo. Allí ingresan en escena las representaciones sociales. Representaciones que no son aisladas pues sus matrices de sentido se construyen enmarcadas en un espacio social que es configurado por, y a la vez, configura a las instituciones organizadas. En esos devenires transcurren las juventudes cuyos procesos de socialización son marcados por los rituales y códigos simbólicos propios de dichas instituciones. Tal como podemos notar en el primer párrafo utilizamos el plural en el concepto demarcado por el paréntesis- para notar que no existe un solo modo de ser joven y por consiguiente de transitar los recorridos subjetivos e institucionales.

\section{Perfiles juveniles}

Identificando que en la juventud se hallan presente esos crisoles de experiencias en donde se presentan diferentes condiciones sociales, parece relevante detenernos en las particularidades de los que integraron nuestra muestra. Luego de ello podremos advertir su incidencia en la constitución de las representaciones sociales sobre violencia de género.

Entre los datos que fueron consultados en los cuestionarios se encuentran: edad-estrato socio económico-identidad de género autopercibida. De tales datos podemos apreciar que la edad mínima se concentra en 19 y la máxima en 27 años. A partir de ello se obtiene una media de 22.63. Tal como hemos señalado anteriormente, consideramos a la juventud como una experiencia vital en la que converge una diversidad de condiciones y por consiguiente no es posible anclarla sólo a criterios etarios. Anaqueles completos de las bibliotecas en dicho campo de estudios han descripto la relevancia de no circunscribir la juventud solamente al componente material que aporta la edad. Para reflejar estos aspectos acudiremos a algunos referentes tradicionales y a otros recientes. Margulis y Urresti (1996) marcan una clara distinción respecto de la propuesta bourdieusiana contenida en La Juventud no es más que una palabra (2002), aunque actualmente admite matices, por cuanto no sólo se trata de una construcción simbólica sino también incorpora un aspecto material. Por ello -los investigadores argentinos antes citados- agregan el estudio de las desigualdades sociales en su cruce con la edad para reflejar que la juventud no sólo supone un estilo o estética particular, sino también un componente material cronológico que proviene de la edad y marca la distancia respecto del 
fin de la existencia humana. Asimismo, el concepto desigualdades sociales muestra su interrelación con el sector social de pertenencia y el género, como así también con otras condiciones vinculadas a diferentes capitales, tales como las capacidades/discapacidades, estéticas hegemónicas, trayectorias vitales, procedencia, etcétera. Como jóvenes de sectores populares no experimentan la edad del mismo modo que los de sectores medios y lo mismo sucede con las mujeres. Para utilizar las expresiones de Margulis y Urresti, podemos aseverar que la moratoria social y la vital se alteran sustancialmente a partir de la incidencia de los diacríticos subjetivos antes mencionados. A modo de ejemplo: la pertenencia a un estrato socioeconómico bajo cercena la posibilidad de acceso a determinados consumos que son identificados con el mundo juvenil. Por su parte, la maternidad en las mujeres también modifica la experiencia vital juvenil obligando a transitar muchas veces -tal como hemos relevado en Becher (2018a)- por una etapa cuasi esquizoide en la que pendulan entre permanecer en la etapa joven o transitar el pasaje hacia la adulta (4). En la actualidad han obtenido mayor presencia los enfoques generacionales. Si bien el concepto de generación ya estaba presente en la propuesta de Margulis y Urresti, ha ido adquiriendo mayor centralidad en tiempos recientes. Tal propuesta reconoce antecedentes clásicos en autores tales como Ortega y Gasset (1966) y Mannheim (1993). En tal sentido, Leccardi y Feixa (2011) proponen un recorrido por tales perspectivas para identificar el modo en que han sido apropiadas por estudios recientes sobre juventudes. En un relevamiento previo pudimos advertir que en Argentina tales perspectivas redundan en torno a dos conceptos centrales: socialización y subjetividad e identidad social. De modo tal que para algunos investigadores/as la experiencia generacional supone compartir procesos de socialización -en donde podemos reconocer la influencia de Mannheim- mientras otros reconocen en dicha experiencia -bajo la influencia no explicitada de Abrams (1982)- constituciones subjetivas e identidades sociales compartidas (5). En el dato sobre sectores sociales de pertenencia el predominante es el que corresponde a los sectores medios (treinta casos) mientras ocho se agrupan en bajo y sólo dos en alto. Ello nos muestra las posibilidades que estos jóvenes tienen de acceso a recursos y consumos. De hecho actualmente se plantea la posibilidad de superar la tradicional diferenciación, lo cual no implica renunciar a ella, entre clases sociales por cuanto en la constitución de un sector social de pertenencia no sólo se ponen en juego recursos económicos sino también sociales y simbólicos (6). El concepto mismo de capitales propuesto por Bourdieu (2007) -que se constituyen al interior de un campo determinado- parece ser retomado por estas nuevas miradas para explicar que un sector social se conforma a partir del entretejido que integran las relaciones sociales y el acceso a determinados consumos. Desde hace muchos años, Bauman (2014) en sus diferentes aportes ha insistido en los efectos subjetivos y subjetivantes del 
mercado de consumo. A partir de ello, el autor propone la distinción entre ciudadanos de pleno derecho -quienes pueden satisfacer sus necesidades- y ciudadanos fracasados que son quienes no logran el acceso a las propuestas del mercado. Es subjetivo por cuanto tiene un profundo enraizamiento en las cogniciones y en los comportamientos que distinguen entre uno y otro tipo de ciudadano. Con todo ello queremos reflejar que el concepto de sector social no sólo nos brinda el dato sobre la situación económica de las/os jóvenes sino también sobre sus relaciones sociales y estilos o estéticas.

El último dato que consultamos para terminar de delinear los perfiles juveniles es el referido a la identidad de género autopercibida. Utilizamos esa expresión por cuanto permite reflejar aunque pueda resultar redundante- dos conceptos que se hallan localizados en la definición de género: el de identidad y el de autopercepción. Resultan redundantes por cuanto -tal como se afirma desde la psicología social- la autopercepción es constitutiva de la identidad personal. Sin embargo nos parece que la reiteración puede ser de utilidad por cuanto apela a conceptos que en los últimos tiempos se han ido instalando en el discurso social sobre el género. Aunque debemos admitir que tal consulta fue la que más inquietudes generó entre las/os jóvenes que completaron el cuestionario. Los datos recabados muestran que quienes respondieron se autoperciben en un total de 31 casos con el género femenino mientras nueve lo hacen con el masculino. Asimismo quienes respondieron sobre sus identidades de género también lo hicieron -de modo espontáneo y sin que fuera consultado- sobre sus preferencias sexuales. De modo que luego de señalar femenino o masculino colocaron, en algunos casos, heterosexual. Ello nos aporta como dato que de modo predominante la muestra estuvo constituida por jóvenes cuyas identidades de género coinciden con los comportamientos sexuales que son atribuidos socialmente a las mismas.

Sin dudas tales datos van a iluminar las reflexiones posteriores sobre las representaciones sociales acerca de la violencia de género.

\section{Representaciones sociales: ajustando la lente para una primera mirada}

\section{Significados}

Apelando al método de asociación libre de palabras pudimos identificar aquellos significantes que las juventudes que integraron la muestra asociaron al de violencia de género. Asimismo tal como aclaramos anteriormente- a partir de concebir a las juventudes como una experiencia vital anclada en condiciones sociales y simbólicas particulares, nos parece importante presentar estos significantes relacionados con el género y el estrato socioeconómico. 
Del cruce entre significantes y género surge que las mujeres asocian violencia de género con golpes (nueve casos); patriarcado (siete casos); violencia física (seis casos); violencia psicológica (cuatro casos); insultos (dos casos); violencia sexual (un caso) y femicidio (un caso). Por su parte quienes se identifican como varones colocan en primer lugar patriarcado (cuatro casos) y siguen: golpes (tres casos) y violencia psicológica (dos casos) (ver Tabla 1 y Figura 1).

\begin{tabular}{|c|c|c|c|c|c|c|c|c|c|c|}
\hline \multicolumn{11}{|l|}{ Recuento } \\
\hline & & \multicolumn{8}{|c|}{ ASOCIACION PALABRAS CON VIOLENCIA } & \multirow[b]{2}{*}{ Total } \\
\hline & & Fisica & Golpes & Sexual & Psicológica & Femicidio & Violación & Insultos & Patriarcado & \\
\hline \multirow{2}{*}{$\begin{array}{l}\text { IDENTIDAD DE GÉNERO } \\
\text { AUTOPERCIBIDA }\end{array}$} & Femenino & 6 & $\overline{9}$ & 1 & 4 & 1 & 1 & 2 & 7 & 31 \\
\hline & Masculino & 0 & 3 & 0 & 2 & 0 & 0 & 0 & 4 & 9 \\
\hline Total & & 6 & 12 & 1 & 6 & 1 & 1 & 2 & 11 & 40 \\
\hline
\end{tabular}

Tabla 1. Identidad de género y significantes asociados. Fuente: elaboración propia con uso del programa SPSS.

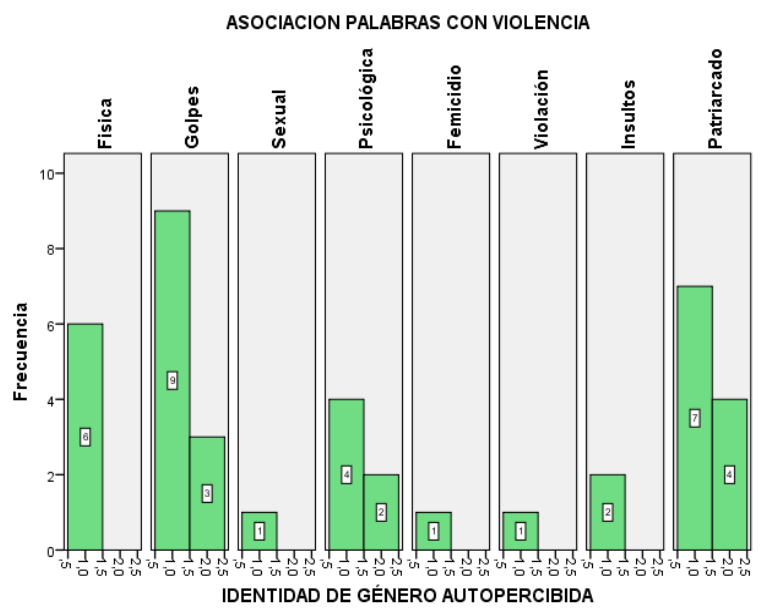

Figura 1. Identidad de género y significantes asociados. Fuente: elaboración propia con uso del programa SPSS.

Las jóvenes provenientes de estratos socioeconómicos bajos representaron violencia de género con su clasificación en física (tres casos) y lo mismo sucede con quienes pertenecen a sectores medios (doce casos) en donde se concentra la mayoría de la muestra. Las integrantes del colectivo socio generacional que pertenecen a sectores altos -sólo dos casos- utilizaron significantes tales como insultos y patriarcado. Entre quienes se reconocen como varones de sectores bajos, aparece de modo predominante el significante patriarcado. En los estratos 
medios su representación se vincula con golpes. No se registraron varones que se identifiquen como pertenecientes a sectores altos (ver Tabla 2 y Figura 2).

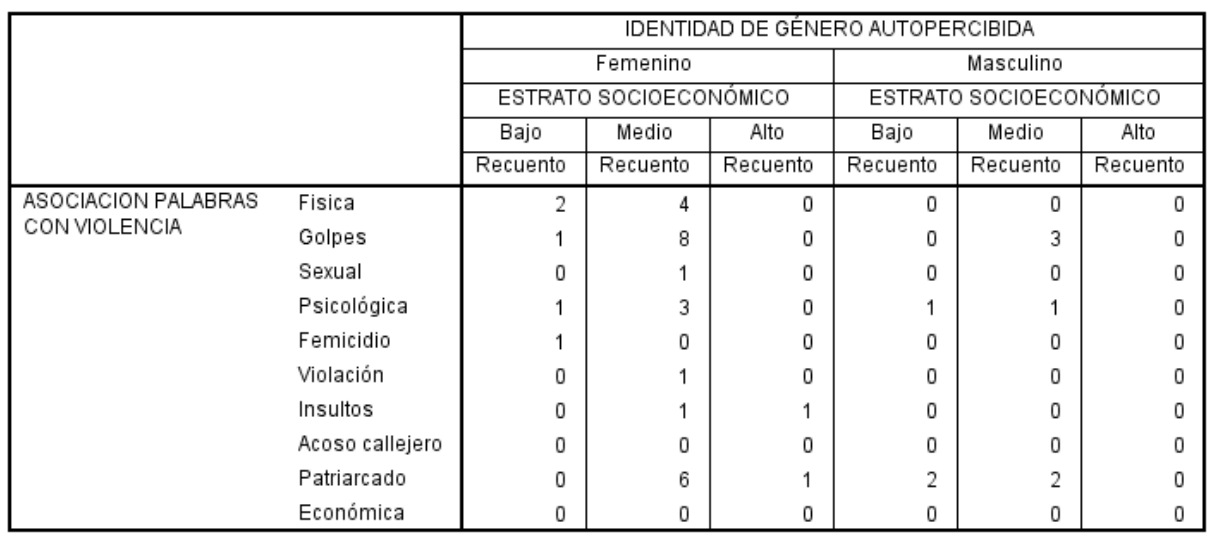

Tabla 2: estrato socioeconómico y significantes asociados. Fuente: elaboración propia con uso del programa SPSS.

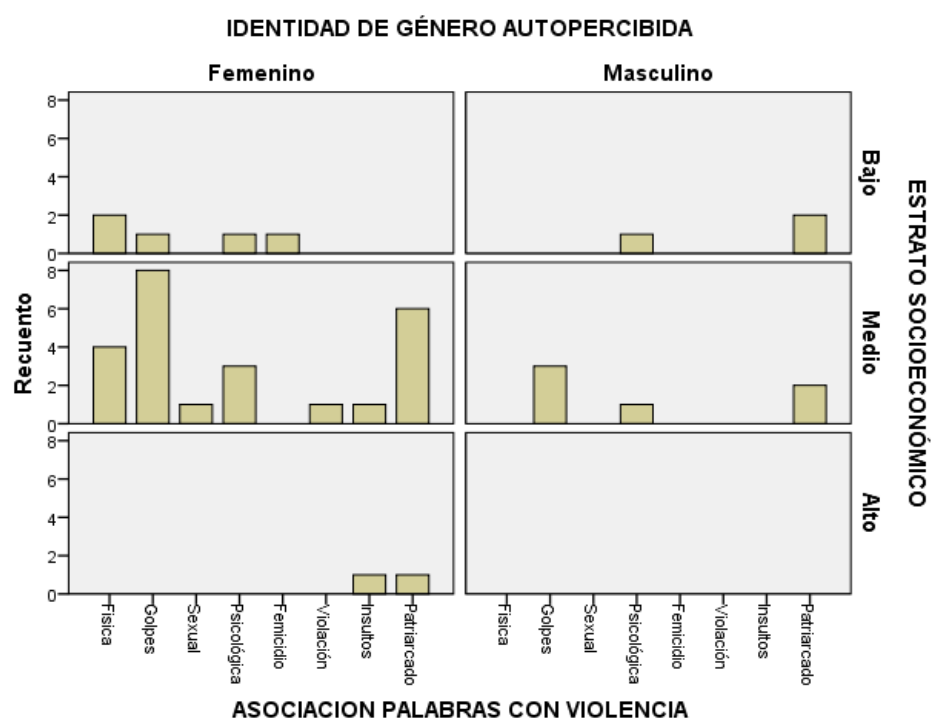

Figura 2: estrato socioeconómico y significantes asociados. Fuente: elaboración propia con uso del programa SPSS.

Tanto entre jóvenes varones y mujeres -incluso provenientes de diferentes estratos socioeconómicos- el nodo central de sus representaciones sociales en torno a la violencia de género gira en relación con su tipificación como violencia física. Si sumamos los casos que la vinculan con golpes y propiamente con violencia física suman un total de 15 sobre 40 . En relación con el núcleo central de las representaciones, tal como afirman Petracci y Kornblit 
(2004): "Estos elementos cognitivos... se encuentran muy ligados a la memoria colectiva y a la historia del grupo y son, por ende, resistentes a los cambios y poco sensibles a las modificaciones del contexto social inmediato" (p. 94). Cada época, tal como señalaba Foucault (1988), es portadora de un discurso que se presenta como dominante respecto de otros. En esos discursos se entraman las redes de poder que como tales admiten resistencias. Sin dudas, un discurso social dominante en materia de violencia de género ha sido el que históricamente la ha circunscripto a su tipificación como física. Aunque tampoco es menos cierto el considerable incremento y constante presencia que su manifestación extrema -el feminicidio- ha tenido en tiempos contemporáneos argentinos. A partir de ello es posible notar la influencia que el espacio social o el contexto va ejerciendo en la configuración de los sentidos sociales. Por consiguiente, las representaciones sociales inciden en el contexto y al mismo tiempo informan sobre él. Tal como dice Araya Umaña: “... las RS contribuyen a construir el objeto del cual son una representación, por lo que este objeto es, en parte, realmente tal y como aparece a través de su representación social (Ibáñez, op.cit)" (2002: 48). El concepto de femicidio o feminicidio ha suscitado diversas discusiones en el campo de las Ciencias Sociales. Sin embargo nos parece importante -tal como plantea Marcela Lagarde (2006) quien adhiere al uso de la noción feminicidio- identificar tal comportamiento con la muerte de mujeres como consecuencia de la misoginia y de la impunidad de parte del Estado. Asimismo, tal como asevera Toledo Vásquez (2009), el concepto de feminicidio incorpora otras conductas delictivas que no necesariamente conducen a la muerte de la mujer sino a situaciones que implican un grave daño a su integridad física, psíquica o sexual. De allí que la negación del acceso al derecho a la interrupción voluntaria del embarazo constituya de parte del Estado una acción que puede ser caracterizada como feminicida. Informes del Ministerio Público fiscal y de asociaciones civiles muestran que entre 1990 y 2013 han desaparecido 3231 mujeres (249 por año). Al mismo tiempo -de acuerdo con datos actuales del Poder Judicial de la República Argentina- en 2017 se cometieron 251 feminicidios, mientras cada 30 horas muere una mujer por tal causa.

Jodelet (1991) señala que existen dos orientaciones metodológicas en el estudio de las representaciones sociales. Una de ellas consiste en el uso de la técnica de asociación de palabras para desentrañar los elementos centrales y periféricos de dichas representaciones. En tal sentido un significante que aparece de modo recurrente -tal como muestran los datos- es el de patriarcado. Es preciso mencionar que estas expresiones se van instalando en el discurso social a partir de la presencia de otros discursos -que van fracturando los dominantes- y que logran permeabilidad en la sociedad. El patriarcado como causa de la violencia de género se encuentra ligado a un contexto social en donde predomina la presencia de los movimientos 
feministas, que -tal como señala de Miguel Álvarez (2000)- son los que deben enfrentarse a un orden social que intenta oprimir a las mujeres y a códigos morales ancestrales. Ya hemos aclarado que para mantener dicho orden social es necesario recurrir a la violencia mediante un proceso bifronte que proviene de sujeciones impuestas por la propia sociedad, y otras, producto de la omisión estatal. El interés por mantener ese orden emana precisamente de la presencia de estructuras patriarcales. Femenías lo denomina paradigma patriarcal y lo define como aquel que interpreta y legitima la violencia apelando a algún rasgo que cataloga como natural e inferiorizante: sexo-género; opción sexual; color de piel; condición de migrante; preceptos religiosos, entre otros. "Es decir, cualquier sociedad otorga el significado de marca de inferioridad (coyuntural o sostenida en el tiempo) a algún rasgo así construido" (2018: 23). Precisamente, la historia muestra que son los movimientos feministas los que han ido logrando constituir líneas de fuga respecto de ese paradigma dominante. Posiblemente en la propuesta butleriana (2007) radique el futuro de estos movimientos al romper con los esencialismos identitarios e incorporar otras demandas a fin de lograr mayor adhesión y performatividad social.

\section{Contextos}

De las 31 jóvenes que integraron la muestra y fueron consultadas, 21 afirman haber padecido alguna situación que representan como de violencia de género. El espacio o contexto que indican como aquel en el cual han padecido ese tipo de situaciones ubica en primer lugar incluso cuando provienen de diferentes estratos socioeconómicos- a las relaciones de pareja (ocho) y continúan: la escuela (cuatro); la universidad (cuatro); espacios públicos (cuatro); ámbito laboral (una) y en el ámbito familiar (una). El número total de lugares mencionados excede la cantidad de casos -31- por cuanto en uno de ellos hubo respuesta múltiple. Asimismo vale aclarar que las jóvenes consultadas transitan principalmente espacios educativos universitarios y pocas de ellas se hallan insertas en empleos.

Los jóvenes varones consultados -si bien fueron pocos casos- afirman haber padecido alguna situación que representan como de violencia de género. El espacio que colocan en primer lugar es el constituido por sus relaciones de pareja y continúan muy de cerca el contexto universitario y los espacios públicos (ver tablas y gráficos). 
Tabla cruzada IDENTIDAD DE GÉNERO AUTOPERCIBIDA *CONTEXTO DE PRODUCCIÓN DE VIOLENCIA

Recuento

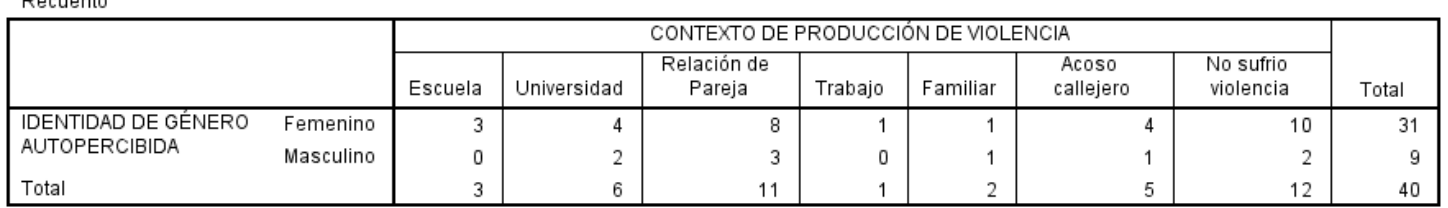

Tabla 3: identidad de género y espacios de violencia. Fuente: elaboración propia por medio del uso del programa SPSS.

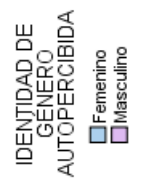

CONTEXTO DE PRODUCCIÓN DE VIOLENCIA

Escuela Relación de Pareja Familiar No sufrio viole

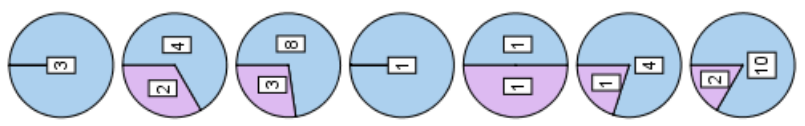

Figura 3: identidad de género y espacios de violencia. Fuente: elaboración propia por medio del uso del programa SPSS.

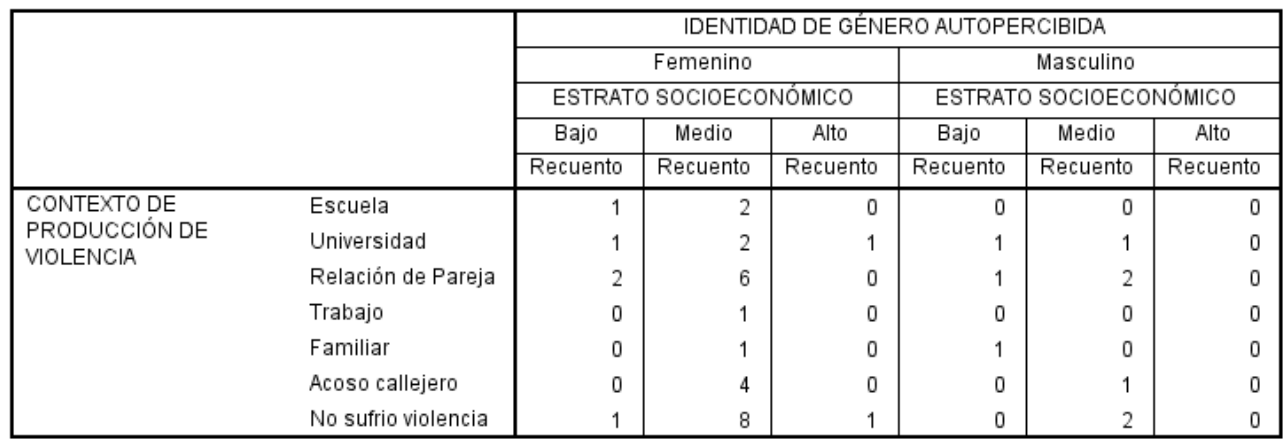

Tabla 4: estrato socioeconómico y espacios de violencia. Fuente: elaboración propia por medio del uso del programa SPSS. 


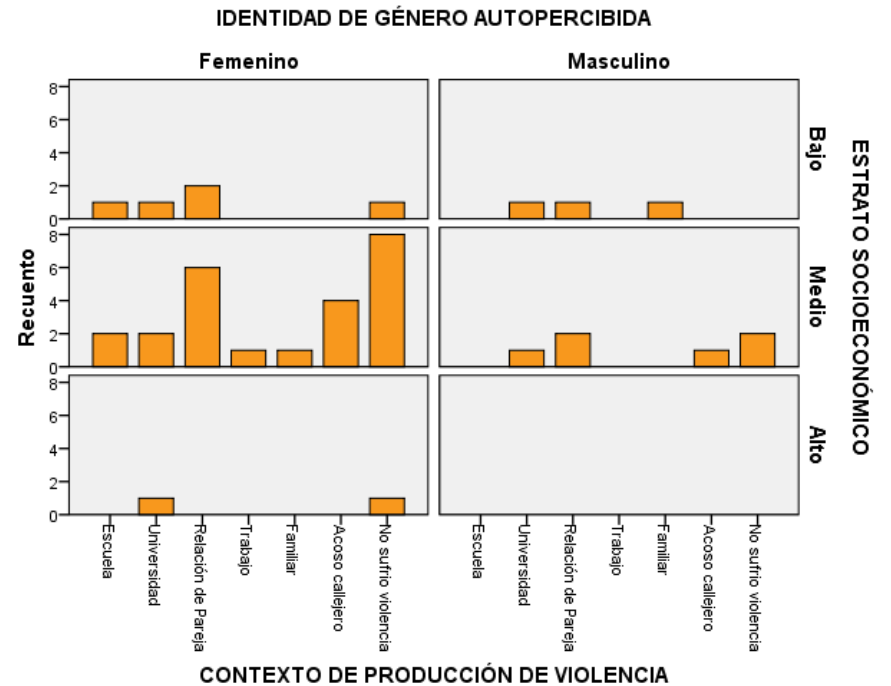

Figura 4: estrato socioeconómico y espacios de violencia. Fuente: elaboración propia por medio del uso del programa SPSS.

Anteriormente dijimos que las instituciones conforman el contexto o espacio social y al mismo tiempo son conformadas por aquel. Si comprendemos por institución a aquel conjunto de pautas o normas que intentan regular el comportamiento subjetivo, podemos incorporar en el concepto todo tipo de espacios -materiales o simbólicos- que tengan ese fin. Estos espacios o instituciones organizadas se encuentran atravesados por relaciones de poder que como tales desde una perspectiva foucaultiana- son asimétricas y admiten resistencias. Una institución, afirma Kaminsky (1998), es un conjunto de prácticas, un dispositivo organizado y orientado a producir comportamientos estereotipados, que están reglados o normados. Se hacen con costumbres y ritos que otorgan sanciones negativas y positivas; son espacios de interacción entre lo imaginario y lo simbólico, donde hay juegos de violencia y contra-discursos. De allí que las jóvenes que identificaron el significante violencia de género con su tipificación en física manifiestan haber padecido situaciones de ese tipo en el marco de sus relaciones de pareja. Existen -como plantean Guedes, Bott, García Moreno y Colombini (2006)- diferentes formas de violencia contra la mujer en donde las más significativas -por sus cifras y modalidades- tal como muestran los estudios mundiales sobre el tema, son las ejercidas por parejas o ex parejas íntimas:

Según cálculos de la Organización Mundial de la Salud (OMS), casi una tercera parte (30\%) de las mujeres que han tenido alguna vez una relación de pareja refiere haber sufrido alguna forma de violencia física o sexual por parte de sus parejas (ONU, 2010: 2). 
A partir de ello, la relación de pareja se constituye como un espacio o contexto en donde suelen predominar situaciones de violencia de género. Allí también las representaciones sociales -que tal como señalaba Araya Umaña- configuran el contexto e informan sobre él, muchas veces producen las condiciones necesarias para el sostenimiento de vínculos violentos con parejas. En este caso nos referimos a los sentidos sociales sobre el amor romántico, que tal como muestran las evidencias empíricas recogidas en Caro Blanco (2008), afectan mayormente a las mujeres. La autora comprueba que el proceso de enamoramiento -en parejas heterosexuales- implica para ellas mayor sufrimiento y dependencia, mientras que no sucede lo mismo con los varones. Ello contribuye a acentuar la construcción asimétrica propia de las relaciones entre el género femenino y el masculino, al mismo tiempo que alimenta el imaginario sobre el amor ideal propio de las culturas occidentales: "La ideología del amor romántico coloca al otro en el centro de nuestro ser. Se piensa que el erotismo, el amor tiene que venir del otro ser, ese dios siempre es el otro, nunca ella misma" (Caro Blanco, 2008: 222). Por ello, la necesidad de aportar a ese imaginario o bien de permanecer en él conduce muchas veces a mantener relaciones poco saludables en donde las mujeres experimentan situaciones de violencia.

Entre las otras instituciones que predominan en los contextos que las/os jóvenes mencionan como aquellos en los que han padecido situaciones de violencia de género colocan los vinculados a la educación: escuela o universidad. Dubet (2010) menciona que los ámbitos educativos son espacios en los que históricamente han predominado las desigualdades como consecuencia -entre otras- del sostenimiento de un programa educativo eclesiástico que los distancia de las mutaciones que se van produciendo en las realidades sociales. De allí que identifica a la modernidad tardía como el momento histórico de clivaje para tales instituciones, por cuanto la masificación escolar trae aparejada una diversidad subjetiva a la cual la educación tiene que dar respuesta. Ese período también implicó un mayor acceso de las mujeres a esas instancias aunque poco se cuestionaba acerca de la necesidad de modificar la gramática escolar a fin de equipar con los varones. Ello pues las instituciones educativas -como espacios predilectos de socialización en valores y normas- suelen producir condiciones para la reproducción de estereotipos de género, limitando expectativas y posibilidades para las mujeres.

García Guevara (2005) afirma que las instituciones educativas son espacios en los que predominan representaciones sociales acerca de lo que se espera de parte del género femenino. A partir de ello circunscriben sus expectativas por cuanto los procesos de socialización que les son ofrecidos tienden a reproducir los lugares -en algunos casos vinculados a las tareas de cuidado- que históricamente les han sido asignados. Precisamente, 
los últimos informes del Comité de la Convención sobre la Eliminación de todas las Formas de Discriminación sobre la Mujer (CEDAW) para la Argentina (2010 y 2016) aseveran la relevancia de superar los estereotipos de género en los ámbitos educativos a fin de favorecer el empoderamiento femenino:

...el Comité expresa su preocupación por la posibilidad de que los estereotipos de género y la influencia de los medios de comunicación lleven a las mujeres a decantarse por ocupaciones sociales tradicionales y limiten sus ventajas comparativas en el mercado laboral (ONU, 2010: 8).

Asimismo, en lo referido a la enseñanza de oficios que en los últimos años se ha realizado por medio de programas sociales -tal como hemos relevado en Becher (2018b)- muestra en la oferta la predominancia de estereotipos sobre ocupaciones femeninas y masculinas. Todas estas observaciones se centran en el plano de los comportamientos que tienen lugar en los ámbitos educativos, sin embargo, para obtener una imagen más completa es necesario revisar lo que sucede a nivel de las cogniciones. Tal como plantea Luce Irigaray -en la revisión que realiza Schutte (1990)- epistemológicamente la mujer siempre ha sido colocada en el espacio correspondiente al objeto de conocimiento, mientras los varones son representados con el sujeto que conoce. Si trasladamos esa premisa -como propone Irigaray- al ámbito del psicoanálisis, principalmente con la fuerte influencia freudiana, es posible reconocer que la mujer suele ser asociada a lo inconsciente -irracional- mientras el varón es significado con la esfera que corresponde a los actos de la consciencia. De modo que tanto a nivel de los comportamientos como de las cogniciones, los ámbitos educativos pueden ser contextos o instituciones hostiles para quienes se autoperciben o identifican con el género femenino.

\section{Reflexiones finales}

El estudio de las representaciones sociales juveniles deviene en un aspecto relevante ya que muestra la influencia que los discursos sociales ejercen en la constitución de esas figuraciones significativas. Los discursos -constitutivos de las representaciones- responden a climas epocales en donde unos $u$ otros significantes logran instalarse. Ello devela que cada época apelando a las enseñanzas foucaultianas antes expuestas- es portadora de un discurso. Asimismo tales discursos configuran representaciones dominantes sobre un significante, logrando tener mayor permeabilidad en un momento histórico determinado. De allí que 
posiblemente en otros momentos la violencia de género no era representada con su tipificación en física o bien como consecuencia del patriarcado.

Sirve realizar indagaciones del tipo de las que se proponen en este artículo -que en este caso constituye un primer acercamiento al tema- por cuanto permiten reflejar el modo en que persisten y se actualizan sentidos sociales en torno a un conjunto de significantes. Los resultados obtenidos muestran que las/os jóvenes que fueron consultados -incluso provenientes de estratos sociales contrastantes- representan la violencia de género predominantemente con su tipificación en física y la anudan con otro elemento presente en la configuración de esos sentidos: el patriarcado.

Podríamos quedarnos lisa y llanamente con estos resultados y no aventurarnos a proponer ninguna lectura. Sin embargo aquel no es el sentido -y la actitud propia- que ha motorizado a las ciencias sociales y a sus actores. Debemos reconocer que tales representaciones se hallan entramadas con un contexto o espacio social y con aspectos biográficos particulares de las juventudes que fueron consultadas. En ese contexto podemos advertir la presencia de movimientos feministas que han logrado instalar un determinado discurso incidiendo sobre las representaciones sociales. De modo tal que en las consultas aparecen sentidos que permiten advertir -más allá de la predominancia- que los jóvenes distinguen distintos tipos de violencia de género e identifican como causales al patriarcado y la exclusión o desigualdad. Sería ingenuo pensar que tales cogniciones sobre el género se encuentran desancladas de un contexto particular. Contexto en el que -tal como hemos aclarado- las instituciones organizadas juegan su papel por cuanto configuran el espacio social y son configuradas por aquel.

En la lectura que propusimos el tema de las instituciones aparece como una constante pues reconocemos su valor como herramienta socializadora en un conjunto de prácticas y significaciones. Sin embargo, tal como lo planteamos al comienzo, nos parece importante, a los fines del análisis, plantear por separado el estudio de significados, en donde el contexto aparece informando e iluminando esas tramas simbólicas, de las instituciones como organizaciones particulares en las que -como afirma Kaminsky (1998)- la violencia psicosocial es parte constitutiva de las mismas. Por tales motivos planteamos la posibilidad de identificar los espacios organizados en los que las/os jóvenes consultados manifiestan haber padecido situaciones de violencia de género. Entre ellos aparecen de modo predominante las relaciones de pareja y las instituciones educativas. Ambos espacios en los que prevalecen relaciones sociales asimétricas de género y ambas instancias en las que las representaciones sociales parecen tener un rol fundamental: marcar las subjetividades como guiones de sentido que conforman una realidad determinada y determinante. En las relaciones de pareja los imaginarios sobre el amor romántico guían y condicionan los comportamientos femeninos en 
detrimento de sus derechos y libertades, obligando a las mujeres a permanecer en relaciones poco saludables que develan tipos diversos de violencias conjugadas. En los ámbitos educativos predominan estereotipos de género que condicionan las expectativas femeninas, limitando su futura inserción en empleos que permitan emanciparlas de los oficios y profesiones que les son socialmente atribuidos.

Finalmente queremos reflejar -pues es un aspecto latente en el recorrido que hemos ido realizando- que nos dedicamos a mostrar y analizar representaciones sociales dominantes sobre violencia de género, lo cual no supone desconocer que en los discursos juveniles relevados emergen otros sentidos, que seguramente en tiempos históricos diferentes irán decantando en dominantes o bien continuarán suturando los caminos de la alternancia y la resistencia. Asimismo, el concepto de representaciones sociales no agota su fertilidad teórica en la propuesta que hemos presentado, lo cual nos motiva a continuar indagando e incorporando nuevas herramientas conceptuales y metodológicas que permitan profundizar esta primera mirada.

\section{Notas}

(1) En la web de la ONG se pueden consultar los datos: http://www.lacasadelencuentro.org/

(2) La violencia con motivos de género fue incorporándose progresivamente en los cuerpos normativos internacionales. Sus primeros antecedentes se ubican en la Conferencia Mundial de los Derechos Humanos -celebrada en Viena en 1993 por la Asamblea General de las Naciones Unidas- que aprobó la Declaración sobre la Eliminación de la Violencia contra la Mujer. Ese documento constituye un hito a nivel internacional ya que se aborda la violencia de género dentro del ámbito de los derechos humanos.

(3) Allí deben tenerse en cuenta otras normativas -tales como la de matrimonio igualitario (Ley 26618 de 2010) o de identidad de género (Ley 26743 de 2012)- que fueron promoviendo transformaciones culturales que derivaron en nuevos activismos y discursos que incidieron sobre las representaciones sociales.

(4) No quiere decir que no suceda con las masculinidades. Sin embargo, se presenta en el caso particular de las mujeres por las tareas de cuidado que trae aparejada la maternidad. A partir de las responsabilidades que imponen tales tareas resulta dificultoso para las subjetividades femeninas permanecer en la etapa juvenil mientras asumen obligaciones que son representadas como propias de la vida adulta.

(5) Mariana Chaves (2006) en su difundido informe sobre investigaciones en juventudes adopta una perspectiva sobre el concepto que lo vincula con los procesos de socialización. Investigaciones posteriores -entre las que se pueden consultar las de investigadores en juventudes del Instituto de Investigaciones Gino Germani de la Universidad de Buenos Aires- acuden al concepto de subjetividad e identidad para explicar la experiencia generacional.

(6) Bonvillani (2018) en su reciente libro Entre el folclore de la fiesta y lo irreparable de la muerte juvenil. La experiencia de la marcha de la gorra describe con claridad el modo en que se constituyen subjetivamente las/os jóvenes de sectores populares que incorpora en su investigación, quienes no sólo se definen a partir de poseer determinados 
recursos económicos sino también desde preferencias musicales y estéticas. Incluso las redes de pares que construyen se vinculan con esos criterios de identificación.

\section{Bibliografía}

Abrams, P. (1982). Historical Sociology. Shepton Mallet: Open Books.

Araya Umaña, S. (2002). Las representaciones sociales. Ejes teóricos para su discusión. Costa Rica: FLACSO.

Aubric, J. C. (1976). Jeux, conflits et représentations sociales. (Tesis doctoral). Université de Provence, Francia.

Bauman, Z. (2014). ¿La riqueza de unos pocos nos beneficia a todos? (Traducido por Alicia Capel Tatjer). Ciudad de Buenos Aires: Paidós.

Becher, Y. (2018a). El devenir femenino juvenil en programas sociales. Ponencia presentada en la 8a․ Conferencia Latinoamericana y Caribeña de Ciencias Sociales y 1ํ Foro Mundial del Pensamiento Crítico, CLACSO, Buenos Aires.

Becher, Y. (2018b). Las juventudes y su vínculo con la burocracia estatal: entre marcas generacionales y redes de sociabilidad. En Castro, G. (Comp.). Militancias y políticas juveniles. Involucramientos sociales en contextos provinciales (pp. 281-319). Buenos Aires: Teseo.

Bonvillani, A. (2016). Algunas pistas para pensar la "juventud" como categoría analítica en procesos investigativos. Estudio, 21, 4-15.

Bonvillani, A. (2018). Entre el folclore de la fiesta y lo irreparable de la muerte juvenil. La experiencia de la Marcha de la Gorra. Buenos Aires: Grupo Editor Universitario.

Bourdieu, P. 1990). La "juventud" no es más que una palabra. En Bourdieu, P. Sociología y cultura. México: Grijalbo.

Bourdieu, P. (2007). El sentido práctico. Buenos Aires: Siglo XXI Editores.

Butler, J. (2007). El género en disputa. El feminismo y la subversión de la identidad. Buenos Aires: Paidós.

Caro Blanco, C. (2008). Un amor a tu medida. Estereotipos y violencia en las relaciones amorosas. Revista de Estudios de Juventud, 83, Universidad Nacional de La Plata, pp. 213-228.

Chaves, M. (2006). Informe. Investigaciones sobre juventudes en Argentina. Estado del arte en Ciencias Sociales. La Plata-Ciudad de Buenos Aires: UNSAM, Ministerio de Desarrollo Social, DINAJU, UNICEF. 
Cook, R. J. y Cusack, S. (2010). Estereotipos de género. Perspectivas legales transnacionales. Colombia: Profamilia.

De Miguel Álvarez, A. (2000). Movimiento feminista y redefinición de la realidad. Ponencia presentada en el Congreso Feminista de Córdoba, España.

Dubet, F. (2010). Crisis de la transición y declive de la institución. Política y sociedad, 47(2), 1525.

Femenías, M. L. (2018). Violencia contra las mujeres: obstáculos para enfrentarla. En Femenías, M. L. y Novoa, S. M. (Coords.). Mujeres en el laberinto de la justicia. Los ríos subterráneos (volumen VI) (pp. 21-36). Rosario: Prohistoria Ediciones.

Foucault, M. (1988). El sujeto y el poder. Revista Mexicana de Sociología, 50(3), Universidad Nacional Autónoma de México, pp. 3-20.

García Guevara, P. (2005). Género, educación y política pública. Revista de Estudios de Género. La Ventana, 21, Universidad de Guadalajara, pp. 70-89.

González Rey, F. (2008). Subjetividad social, sujeto y representaciones sociales. Revista Diversitas-Perspectivas en Psicología, 4(2), 225-243.

Guedes, A. C.; Bott, S.; García Moreno, C. y Colombini, M. (2006). Bridging the gaps: a global review of intersections of violence against women and violence against children. Global Health Action.

Guattari, F. (2006). Subjetividad e historia. En Guattari, F. y Rolnik, S. Micropolítica. Cartografías del deseo (39-147). Madrid: Traficantes de Sueños.

Jodelet, D. (1986). La representación social: fenómenos, conceptos y teoría. En Moscovici, S. (Ed.). Psicología social (vol. II). Barcelona: Paidós.

Jodelet, D. (1991). Representaciones sociales, un área en expansión. En Páez, D.; Sanjuan, C.; Romo, I. y Vergara, A. (Eds.). Sida: imagen y prevención. Madrid: Fundamentos.

Kaminsky, G. (1998). Dispositivos institucionales. Democracia y autoritarismo en los problemas institucionales. Buenos Aires: Lugar Editorial.

Lagarde, M. (2006). Del femicidio al feminicidio. Desde el Jardín de Freud, 6, Universidad Nacional de Colombia, pp. 216-225.

Leccardi, C. y Feixa, C. (2011). El concepto de generación en las teorías sobre la juventud. Última Década, 34, CIDPA, Valparaíso, Chile, pp. 11-32.

Ley 26485 (2009). Ley de protección integral a las mujeres. Ministerio de Justicia y Derechos Humanos, Presidencia de la Nación. Recuperado de http://servicios.infoleg.gob.ar/infolegInternet/anexos/150000-154999/152155/norma.htm Mannheim, K. (1928). El problema de las generaciones. Revista Española de Investigaciones Sociológicas, 62. 
Margulis, M. y Urresti, M. (1996). La juventud es más que una palabra. En Margulis, M. (Ed.). La juventud es más que una palabra. Buenos Aires: Biblos.

Massey, D. (2007). Geometrías del poder y la conceptualización del espacio [conferencia]. Universidad Central de Venezuela.

Maynes, M. J. (2008). Age as a category of historical analysis: history, agency, and narratives of childhood. The Journal of the History of Childhood and Youth, 1(1), The John Hopkins University Press, pp. 114-124.

Moscovici, S. (1993). Razón y cultura. En Moscovici, S. y Barriga, S. Ante la nueva Europa. Madrid: EUDEMA.

Olsen, F. (2000). El sexo del derecho. En Ruiz, A. E. C. (Comp.). Identidad femenina y discurso jurídico (pp. 25-42). Buenos Aires: Biblos.

Organización de Naciones Unidas (ONU) (2016). Observaciones finales sobre el séptimo informe periódico de la Argentina. Comité de la Convención sobre la Eliminación de todas las Formas de Discriminación contra la Mujer (CEDAW). Recuperado de https://bit.ly/2qGwflH

Organización de Naciones Unidas (ONU) (2010). Observaciones finales sobre el sexto informe periódico de la Argentina. Comité de la Convención sobre la Eliminación de todas las Formas de Discriminación contra la Mujer (CEDAW). Recuperado de https://bit.ly/2PxIClA

Ortega y Gasset, J. (1923). La idea de las generaciones. En El tema de nuestro tiempo. Obras completas. Madrid: Revista de Occidente.

Petracci, M. y Kornblit, A. L. (2004). Representaciones sociales: una teoría metodológicamente pluralista. En Kornblit, A. L. (2004). Metodologías cualitativas en ciencias sociales (pp. 91-111). Buenos Aires: Editorial Biblos.

Saintout, F. (2007). Jóvenes e incertidumbres. Percepciones de un tiempo de cambios: familia, escuela, trabajo y política. (Tesis de doctorado). FLACSO, Sede Académica Argentina, Buenos Aires. Recuperado de http://repositorio.flacsoandes.edu.ec/handle/10469/1048\#.VdaZaLJ_Oko

Schutte, O. (1990). Irigaray y el problema de la subjetividad. Hiparquia, III. Asociación de Mujeres Argentinas en Filosofía, Universidad Nacional de La Plata.

Toledo Vásquez, P. (2009). Feminicidio. México: Naciones Unidas. 